\title{
A Dual-Band SiGe HBT Frequency-Tunable and Phase-Shifting Differential Amplifier Employing Varactor-Loaded, Stacked LC Resonators
}

\author{
Kazuyoshi Sakamoto and Yasushi Itoh \\ Graduate School of Electrical and Information Engineering, Shonan Institute of Technology, 1-1-25 Tsujido-Nishikaigan, Kanagawa, \\ Fujisawa 251-8511, Japan \\ Correspondence should be addressed to Yasushi Itoh, itoh@elec.shonan-it.ac.jp
}

Received 7 July 2012; Accepted 24 September 2012

Academic Editor: Juan Carlos Bohórquez Reyes

Copyright $\odot 2012$ K. Sakamoto and Y. Itoh. This is an open access article distributed under the Creative Commons Attribution License, which permits unrestricted use, distribution, and reproduction in any medium, provided the original work is properly cited.

\begin{abstract}
A dual-band SiGe HBT frequency-tunable and phase-shifting differential amplifier has been developed for the future active phased array antennas with a multiband, multibeam, and multitarget tracking operation. The amplifier uses varactor-loaded, stacked LC resonators in the design of the output circuit in order to provide frequency-tunable and phase-shifting capabilities for dual frequencies. By utilizing the varactor-loaded LC resonator, which has a variable resonant frequency and a large insertion phase variation, frequency-tunable and phase-shifting performances become available. Moreover, by using the stacked configuration, the frequency and insertion phase can be varied independently for dual frequencies. A dual-band SiGe HBT differential amplifier has achieved a lower-frequency tuning range of 0.56 to $0.7 \mathrm{GHz}$ for a higher fixed frequency of $0.97 \mathrm{GHz}$ as well as a higherfrequency tuning range of 0.92 to $1.01 \mathrm{GHz}$ for a lower fixed frequency of $0.63 \mathrm{GHz}$. A lower-frequency phase variation of $99^{\circ}$ and a higher-frequency phase variation of $90.3^{\circ}$ have been accomplished at 0.63 and $0.97 \mathrm{GHz}$, respectively. This is the first report on the dual-band differential amplifier with frequency-tunable and phase-shifting capabilities.
\end{abstract}

\section{Introduction}

Recent and future wireless systems require a wide range of data rates over several frequency bands, and thus adaptive and reconfigurable transceivers become necessary to support high performance and flexibility [1]. Meanwhile, microwave and millimeter-wave sensors and radar systems require multiband, multibeam scanning phased array antennas as well as a multi-target tracking capability [2]. To address these requirements, multifunction capabilities are strongly required. Especially multiband amplification, frequency tunability, gain and phase control are crucial for realizing adaptive and reconfigurable phased array systems. Most of the traditional multiband amplifiers, however, provide a single function [3]. Meanwhile, the authors have presented multiband amplifiers with frequency-tunable as well as gain control capabilities for bandpass and bandstop types $[4,5]$. To enhance the operational capability, a novel design approach is proposed in this paper for the multiband low-noise differential amplifier with frequencytunable and phase-shifting capabilities. In order to realize both frequency-tunable and phase-shifting performances, varactor-loaded LC resonators are used in the design of the output circuit of the differential amplifier. LC resonators are widely used in the reflection type phase shifter because they provide a full $360^{\circ}$ phase shift [6-8]. With the use of the parallel LC resonator in the output shunt circuit, frequency-tunable and phase-shifting performances with high gain can be achieved at around resonant frequencies. In addition, by cascading the parallel LC resonators in a stacked form, frequency and insertion phases can be varied independently for multiple frequencies. As a multiband phase shifter, the resonator-switching or diplexer method has been proposed for the loaded-line type [9], reflection type [10], and LPF/HPF type [11]. These phase shifters, however, produce additional losses due to the switch or diplexer. 
Since the stacked LC resonator does not require additional switches or diplexers, a low insertion loss and a large phase variation can be expected. To make sure the usefulness of the novel design approach proposed in this paper, the dualband SiGe HBT differential amplifier with frequency-tunable and phase-shifting capabilities has been actually designed, fabricated, and tested.

\section{Circuit Design}

A schematic diagram of the dual-band frequency-tunable and phase-shifting differential amplifier is shown in Figure 1. It employs varactor-loaded, stacked LC resonators in the design of the output circuit to achieve frequency-tunable and phase-shifting capabilities for dual frequencies. $L_{1}, L_{2}$, $C_{1}$, and $C_{2}$ are inductors and capacitors consisting of LC resonators. $C_{V 1}$ and $C_{V 2}$ are a variable capacitance of the varactor diode which is controlled by $V_{C 1}$ and $V_{C 2} . C_{C}$ is a decoupling capacitor having a large capacitance value. Resonant frequencies $f_{1}$ and $f_{2}$ are given as follows:

$$
\begin{aligned}
& f_{1}=\frac{1}{2 \pi \sqrt{L_{1}\left(C_{1}+C_{V 1}\right)}}, \\
& f_{2}=\frac{1}{2 \pi \sqrt{L_{2}\left(C_{2}+C_{V 2}\right)}} .
\end{aligned}
$$

When two parallel LC resonators are cascaded in a stacked form, the following bandstop frequency $f_{3}$ is generated. Consider

$$
f_{3}=\frac{1}{2 \pi} \sqrt{\frac{1 / L_{1}+1 / L_{2}}{C_{1}+C_{V 1}+C_{2}+C_{V 2}}} .
$$

Now $f_{1}<f_{2}\left(L_{1} C_{1}>L_{2} C_{2}\right)$ then $f_{3}$ is necessarily sandwiched between $f_{1}$ and $f_{2}$ as

$$
f_{1}<f_{3}<f_{2}
$$

$C_{V 1}$ and $C_{V 2}$ are carefully designed in accordance with the target frequency range and phase-shifting amount. In order to realize $C_{V 1}$ and $C_{V 2}$, several varactor diodes with a fixed capacitance ratio are connected in series or parallel.

A locus of the reflection coefficient $\Gamma_{L}$ of the varactorloaded, stacked LC resonator is graphically shown in Figure 2. As the frequency increases from zero to $f_{1}, \Gamma_{L}$ moves from the short to the open-circuited point $\mathbf{P}$. Since the LC resonator is actually lossy, the point $\mathbf{P}$ has finite impedance. Then as the frequency increases up to $f_{3}, \Gamma_{L}$ moves to the series resonant point $\mathbf{q}$ where the impedance is not ideally short due to the lossy circuit. As the frequency increases from $f_{3}$ to $f_{2}, \Gamma_{L}$ comes back to the point $\mathbf{P}$ again. Finally $\Gamma_{L}$ goes to short. At around the resonant frequencies $f_{1}$ and $f_{2}$, the insertion phase drastically varies from $+180^{\circ}$ to $-180^{\circ}$. Therefore, with the precise control of $V_{C 1}$ and $V_{C 2}$, the insertion phase can be tuned. In addition, at the resonant frequencies $f_{1}$ and $f_{2}$, a peak gain of the amplifier can be obtained. The mechanism is depicted graphically in Figure 3 by using a frequency response of the amplifier.

It is clearly demonstrated that the frequency can be varied with the coarse control of $V_{C 1}$ and $V_{C 2}$ and that the insertion

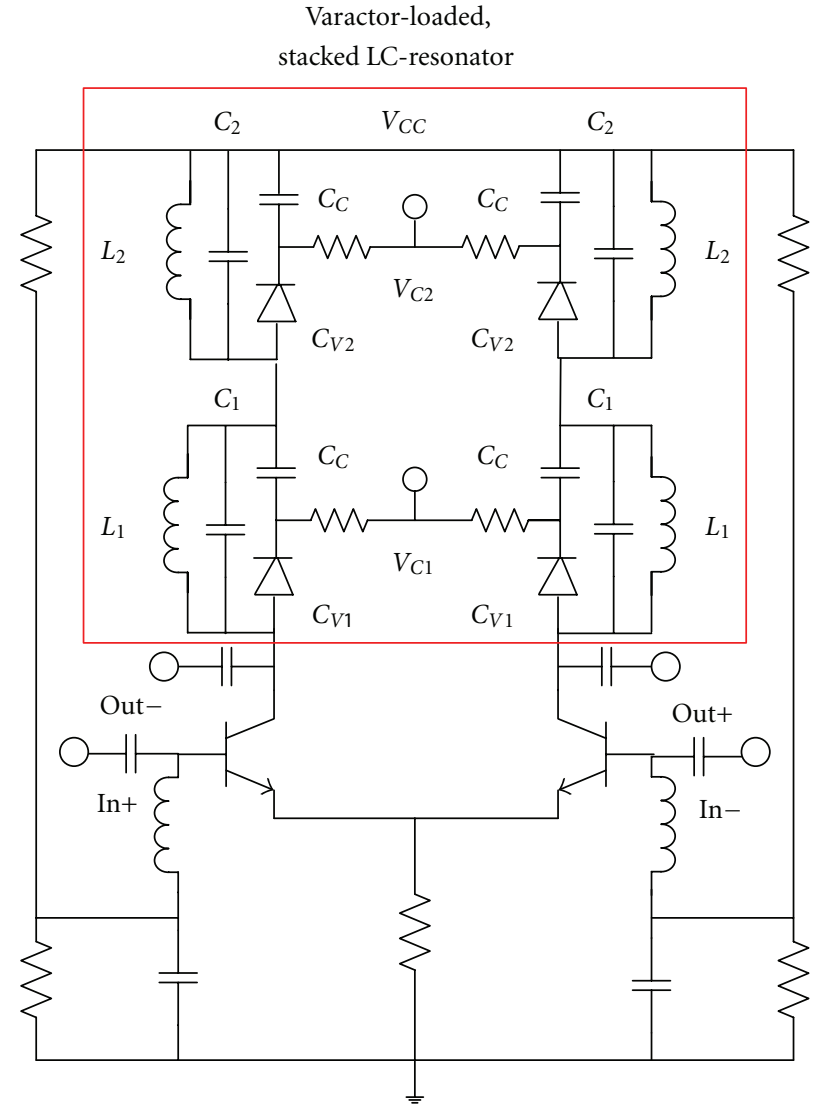

Figure 1: Schematic diagram of the dual-band frequency-tunable and phase-shifting differential amplifier.

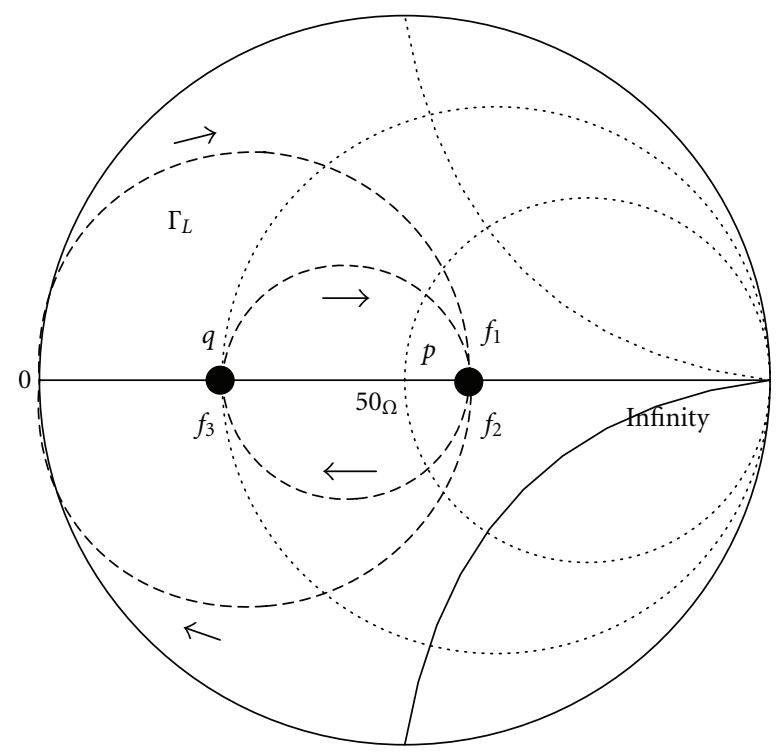

FIgURE 2: Locus of the reflection coefficient $\Gamma_{L}$ of the varactorloaded, stacked LC resonator. 


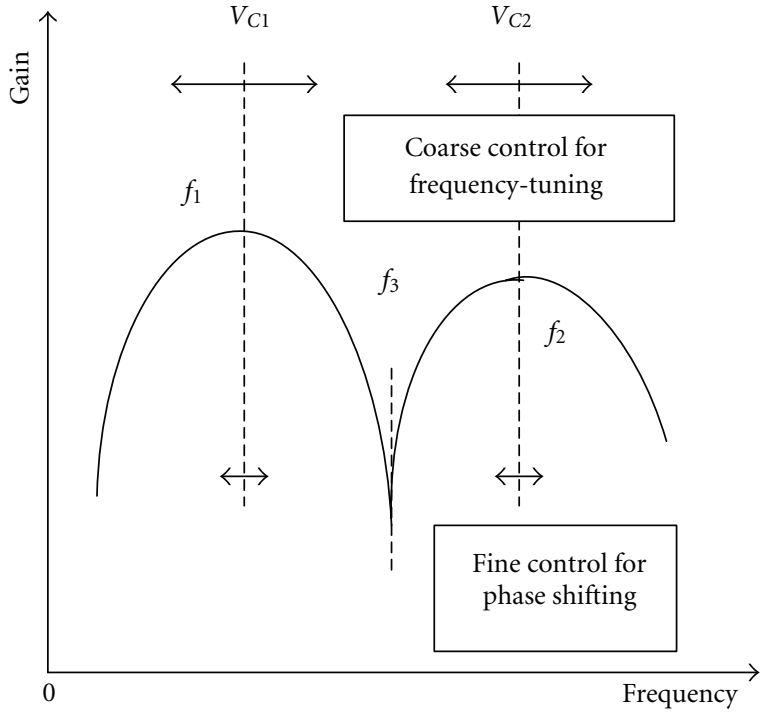

Figure 3: Frequency response of the dual-band frequency-tunable and phase-shifting differential amplifier.

phase can be also varied with the fine control of $V_{C 1}$ and $V_{\mathrm{C} 2} . f_{1}, f_{2}$, and $f_{3}$ are calculated by using from (1) to (3) for the circuit element values listed in Table 1. The circuit element values were chosen to meet the condition of (4). When $C_{V 1}$ varies from 6 to $15 \mathrm{pF}, C_{V 2}$ has a fixed value of $6 \mathrm{pF}$. Meanwhile, when $C_{V 2}$ varies from 6 to $15 \mathrm{pF}, C_{V 1}$ has a fixed value of $15 \mathrm{pF}$. The calculated results are shown in Figures 4 and 5 , respectively. It is noted that $f_{1}$ and $f_{2}$ can be tuned independently and that $f_{3}$ is necessarily sandwiched between $f_{1}$ and $f_{2}$.

\section{Circuit Fabrication}

A photograph of the dual-band frequency-tunable and phase-shifting differential amplifier is shown in Figure 6.

The differential amplifier was fabricated on the FR-4 substrate with a dielectric constant of $4.5 .0 .35 \mu \mathrm{m} \mathrm{SiGe}$ HBT with an $f_{t}$ of $25 \mathrm{GHz}$ (Toshiba MT4S102T), Si varactor diode with a capacitance ratio of 2.5:1 (Toshiba 1SV279), 1005-type chip resistors, and inductors and capacitors are mounted on the substrate by soldering. The circuit size is $16 \times 16 \times 1.2 \mathrm{~mm}^{3}$.

\section{Circuit Performance}

4.1. Frequency-Tunable Performances. Measured gain, input and output return losses for a fixed $V_{C 1}\left(C_{V 1}\right)$ and a variable $V_{C 2}\left(C_{V 2}\right)$ are shown in Figures 7,8 , and 9, respectively. As a coarse tuning, $V_{C 2}\left(C_{V 2}\right)$ was varied from 6 to $18 \mathrm{~V}$ with $1 \mathrm{~V}$ step since the anode voltage is equal to $V_{C C}$ of $6 \mathrm{~V}$. The maximal reverse voltage is $15 \mathrm{~V}$. $f_{2}$ moves from 0.73 to $1.31 \mathrm{GHz}$ for a fixed $f_{1}$ of $0.56 \mathrm{GHz}$. The gain varied from 0 to $7.2 \mathrm{~dB}$ for $f_{2}$ and keeps a constant value of $8.9 \mathrm{~dB}$ for $f_{1}$. $V_{C C}$ and $V_{C 1}$ were $6 \mathrm{~V}$. Since the LC resonators are used in the output circuit, the output return loss is varied with the
TABle 1: Circuit element values of Figure 1.

\begin{tabular}{lc}
\hline Element & Value \\
\hline$L_{1}[\mathrm{nH}]$ & 1 \\
$C_{1}[\mathrm{pF}]$ & 10 \\
$C_{V 1}[\mathrm{pF}]$ & $6-15$ \\
$L_{2}[\mathrm{nH}]$ & 1 \\
$C_{2}[\mathrm{pF}]$ & 3 \\
$C_{V 2}[\mathrm{pF}]$ & $6-15$ \\
$C_{C}[\mathrm{pF}]$ & 1000 \\
\hline
\end{tabular}

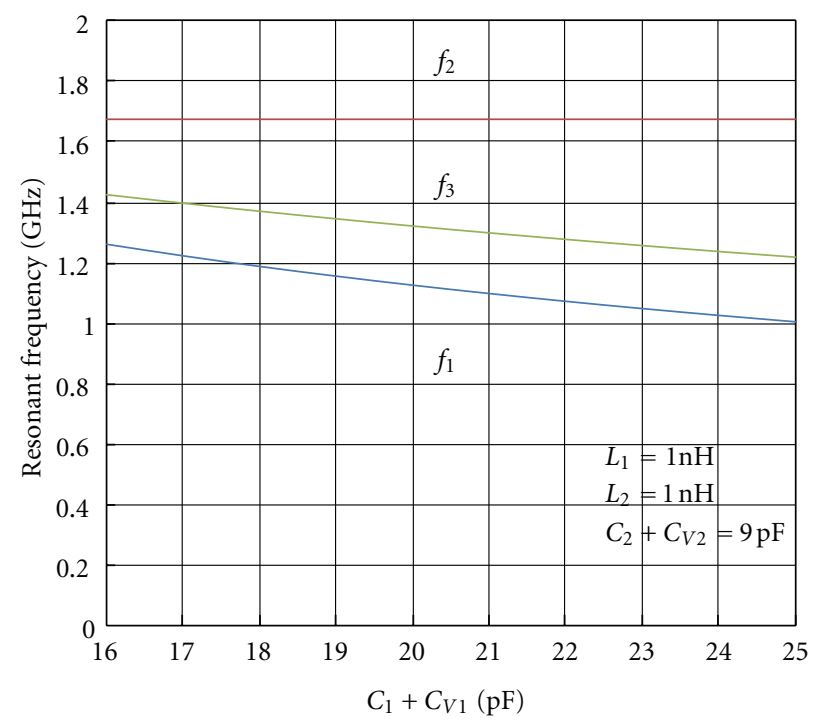

Figure 4: Calculated $f_{1}, f_{2}$, and $f_{3}\left(C_{V 1}\right.$ varies from 6 to $15 \mathrm{pF}$. $C_{V 2}$ has a fixed value of $6 \mathrm{pF}$ ).

resonant frequency, but the input return loss keeps constant. Since the amplifier employed a reflective match approach for low-noise figure, the input and output return losses were less than $10 \mathrm{~dB}$, which can be greatly improved by employing the lossy match approach using resistors [4]. In [4], the input and output return losses can be improved up to greater than $10 \mathrm{~dB}$ across the same frequency band by using the lossy match approach. This approach, however, provides a low gain and a poor noise figure. Thus, the reflective match approach was chosen. Meanwhile, the measured gain, input and output return losses for a variable $V_{C 1}\left(C_{V 1}\right)$ and a fixed $V_{C 2}\left(C_{V 2}\right)$ are shown in Figures 10, 11, and 12, respectively. In a similar way, $V_{C 1}\left(C_{V 1}\right)$ was varied from 6 to $18 \mathrm{~V}$ with $1 \mathrm{~V}$ step. $f_{1}$ moves from 0.57 to $0.97 \mathrm{GHz}$ and $f_{2}$ from 1.31 to $1.34 \mathrm{GHz}$. The gain varied from 8.7 to $13.1 \mathrm{~dB}$ for $f_{1}$ and 1.3 to $6.5 \mathrm{~dB}$ for $f_{2} . V_{C C}$ and $V_{C 2}$ were $6 \mathrm{~V}$. If the varactor-loaded LC resonator shows an ideally open-circuited performance, $f_{1}$ and $f_{2}$ can be controlled independently. But actually the gain and frequency shift at $f_{2}$ was affected to a certain extent by controlling $V_{C 1}\left(C_{V 1}\right)$. This is because the parasitic resistance and low-Q factor of the varactor diode $\left(C_{V 1}\right)$ have made a serious effect on the gain and frequency at $f_{2}$. Therefore, $V_{C 2}\left(C_{V 2}\right)$ in addition to $V_{C 1}\left(C_{V 1}\right)$ must be adjusted simultaneously for keeping $f_{2}$ constant. Moreover, 


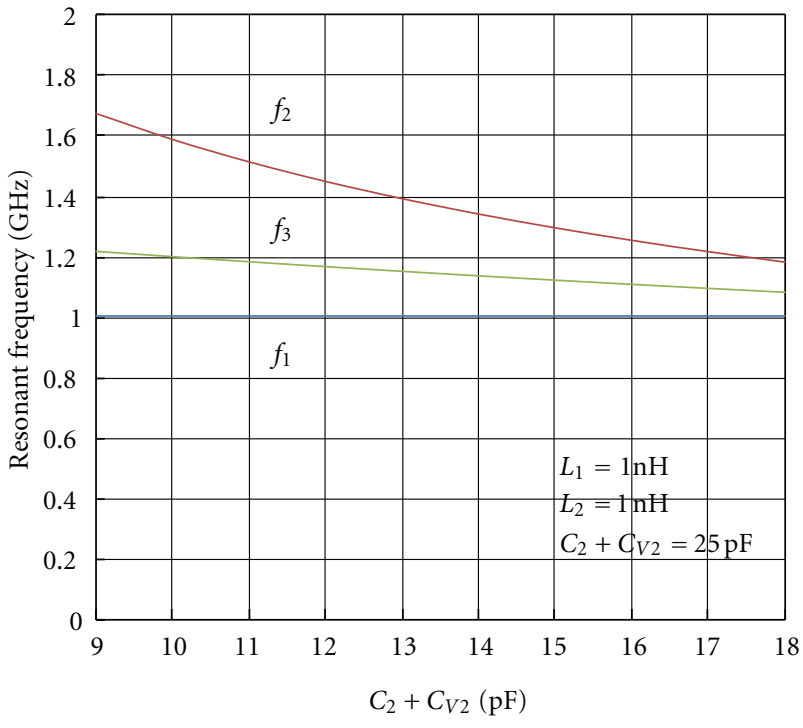

Figure 5: Calculated $f_{1}, f_{2}$, and $f_{3}\left(C_{V 2}\right.$ varies from 6 to $15 \mathrm{pF}$. $C_{V 1}$ has a fixed value of $15 \mathrm{pF}$ ).

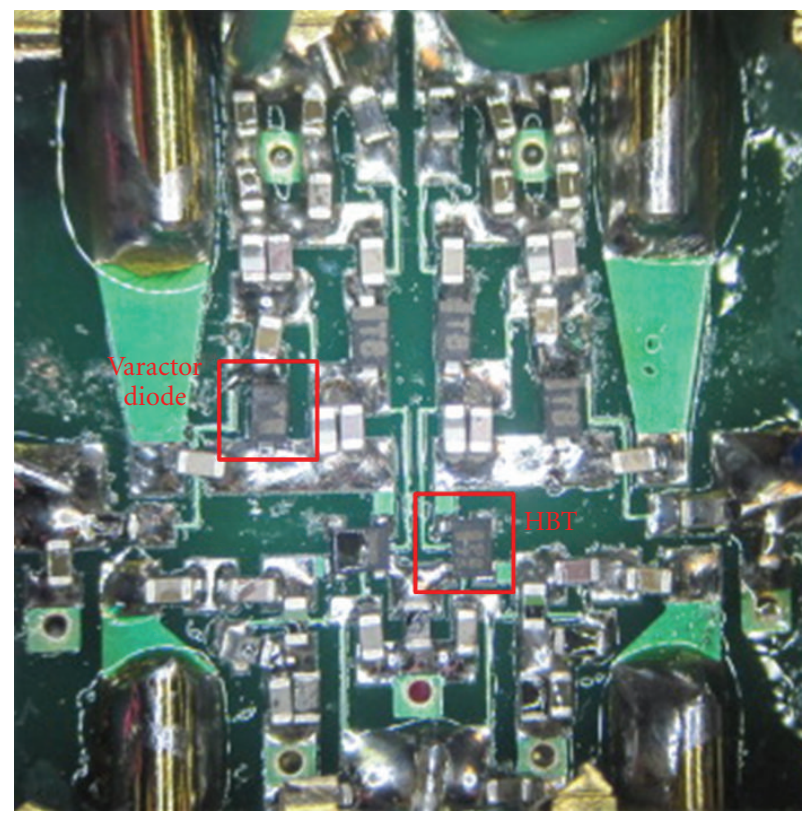

Figure 6: Photograph of the dual-band frequency-tunable and phase-shifting differential amplifier: $16 \times 16 \times 1.2 \mathrm{~mm}^{3}$.

as $f_{1}$ comes close to $f_{2}$, the bandwidth at $f_{1}$ becomes wider, and the gain decreases in Figure 10. This is considered to be the same reason of the parasitic resistance and low-Q factor of the varactor diode. In Figures 7 and 10, the 3rd gain peaking can be seen around $1.6 \mathrm{GHz}$. This is mainly due to the fact that the LC resonator in Figure 1 shows a dual-band resonation due to the lead inductance of the varactor diode.

4.2. Phase-Shifting Performances. Measured phase variations for a fixed $V_{C 1}\left(C_{V 1}\right)$ and a variable $V_{C 2}\left(C_{V 2}\right)$ are shown in Figure 13. As a fine tuning, $V_{C 2}\left(C_{V 2}\right)$ was varied from 10

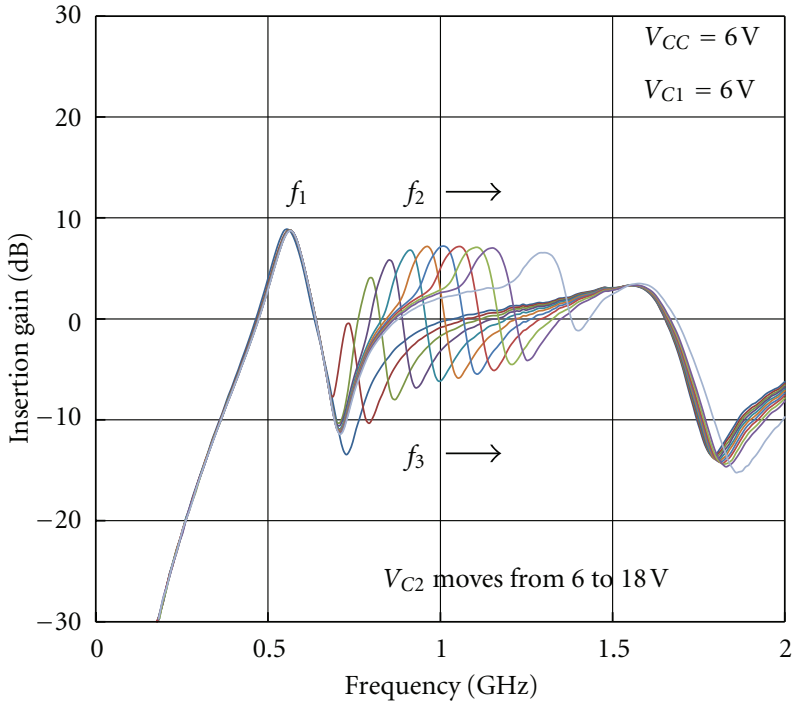

Figure 7: Measured gain for a fixed $V_{C 1}\left(C_{V 1}\right)$ and a variable $V_{C 2}\left(C_{V 2}\right)$.

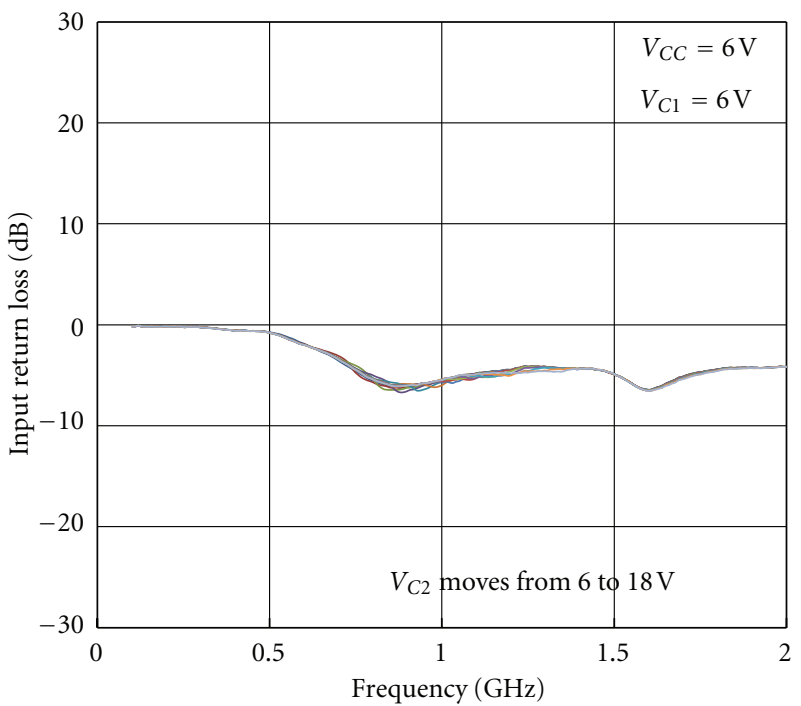

Figure 8: Measured input return loss for a fixed $V_{C 1}\left(C_{V 1}\right)$ and a variable $V_{C 2}\left(C_{V 2}\right)$.

to $12 \mathrm{~V}$ with $0.2 \mathrm{~V}$ step. $f_{2}$ moves from 0.92 to $1.01 \mathrm{GHz}$ for a fixed $f_{1}$ of $0.63 \mathrm{GHz}$. The phase variations at $0.97 \mathrm{GHz}$ and $0.63 \mathrm{GHz}$ were $90.3^{\circ}$ and $2.5^{\circ}$, respectively. $V_{C C}$ and $V_{C 1}$ were $6 \mathrm{~V}$ and $7 \mathrm{~V}$, respectively. The gain variation, however, was $7 \mathrm{~dB}$ at $0.97 \mathrm{GHz}$. The gain variation can be improved by using a lossy match approach, which in turn degrades a phase-shifting amount; that is, there exists a design tradeoff between phase and gain variations. As one approach to address this problem, an additional variable gain amplifier (VGA) with a constant phase is utilized to compensate for the gain variation. Meanwhile, the measured phase variations for a fixed $V_{C 2}\left(C_{V 2}\right)$ and a variable $V_{C 1}\left(C_{V 1}\right)$ are shown in Figure 14. In a similar way, $V_{C 1}\left(C_{V 1}\right)$ was varied from 6 to $8 \mathrm{~V}$ with $0.2 \mathrm{~V}$ step. $f_{1}$ moves from 0.56 to $0.7 \mathrm{GHz}$ for a fixed 


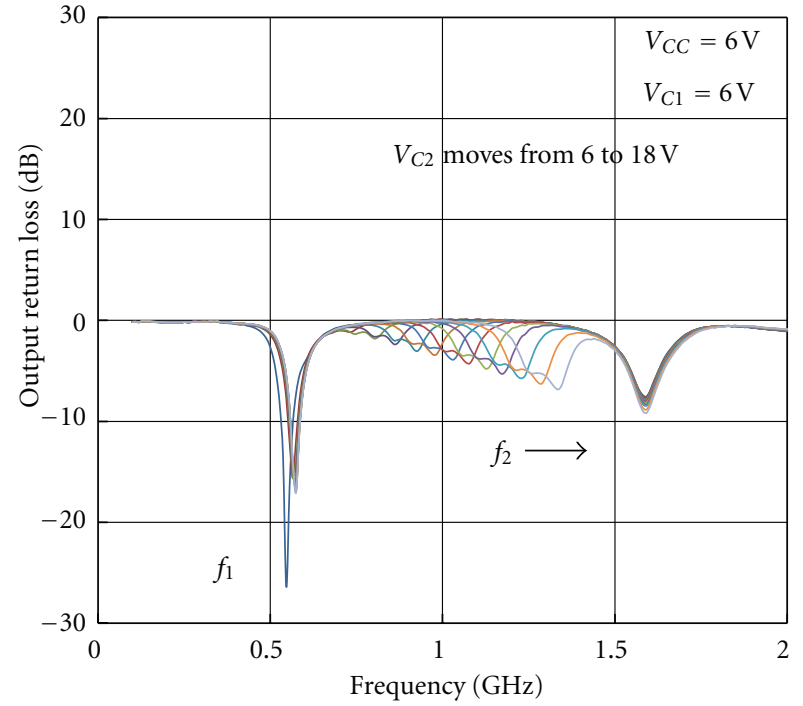

Figure 9: Measured output return loss for a fixed $V_{C 1}\left(C_{V 1}\right)$ and a variable $V_{C 2}\left(C_{V 2}\right)$.

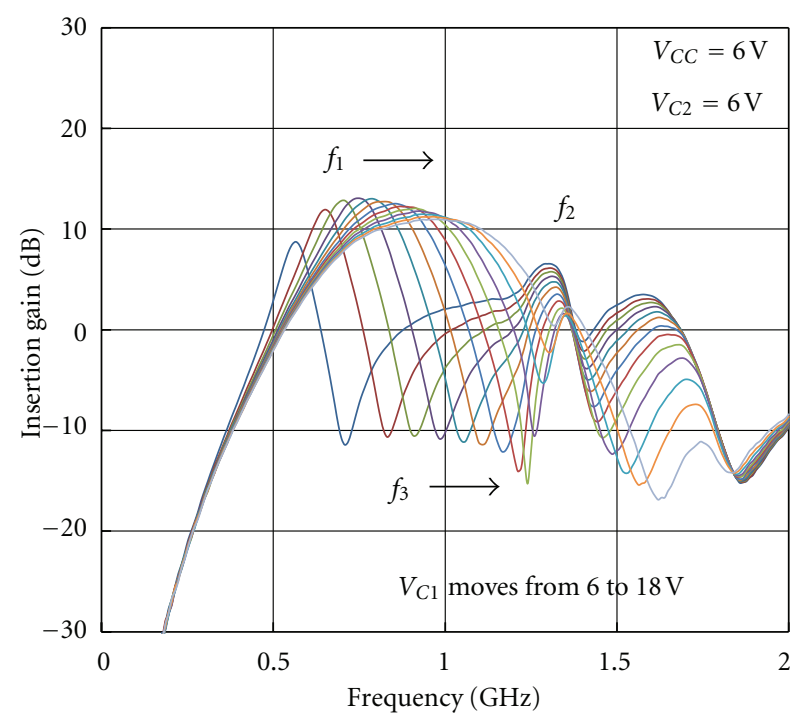

Figure 10: Measured gain for a fixed $V_{C 2}\left(C_{V 2}\right)$ and a variable $V_{C 1}\left(C_{V 1}\right)$.

$f_{2}$ of $0.97 \mathrm{GHz}$. The phase variation at $0.63 \mathrm{GHz}$ was $99^{\circ}$. The phase variation at $0.97 \mathrm{GHz}$, however, was less than $2.1^{\circ}$. $V_{C C}$ and $V_{C 2}$ were $6 \mathrm{~V}$ and $11 \mathrm{~V}$, respectively.

4.3. Noise Figure Performances. Noise figure performances of the dual-band frequency-tunable and phase-shifting differential amplifier have been measured. The measured noise figures are plotted in Figure 15 for a fixed $V_{C 1}\left(C_{V 1}\right)$ and a variable $V_{C 2}\left(C_{V 2}\right)$ and in Figure 16 for a variable $V_{C 1}\left(C_{V 1}\right)$ and a fixed $V_{C 2}\left(C_{V 2}\right) . V_{C C}, V_{C 1}$, and $V_{C 2}$ were $6 \mathrm{~V}, 7 \mathrm{~V}$ and 9-13 V in Figure 15. Meanwhile, $V_{C C}, V_{C 1}$, and $V_{C 2}$ were $6 \mathrm{~V}$, $5-9 \mathrm{~V}$, and $11 \mathrm{~V}$ in Figure 16. The measured noise figures were less than $4.1 \mathrm{~dB}$ at $0.63 \mathrm{GHz}$ and less than $5.3 \mathrm{~dB}$ at $0.97 \mathrm{GHz}$ in Figure 15. Meanwhile, the measured noise figures were

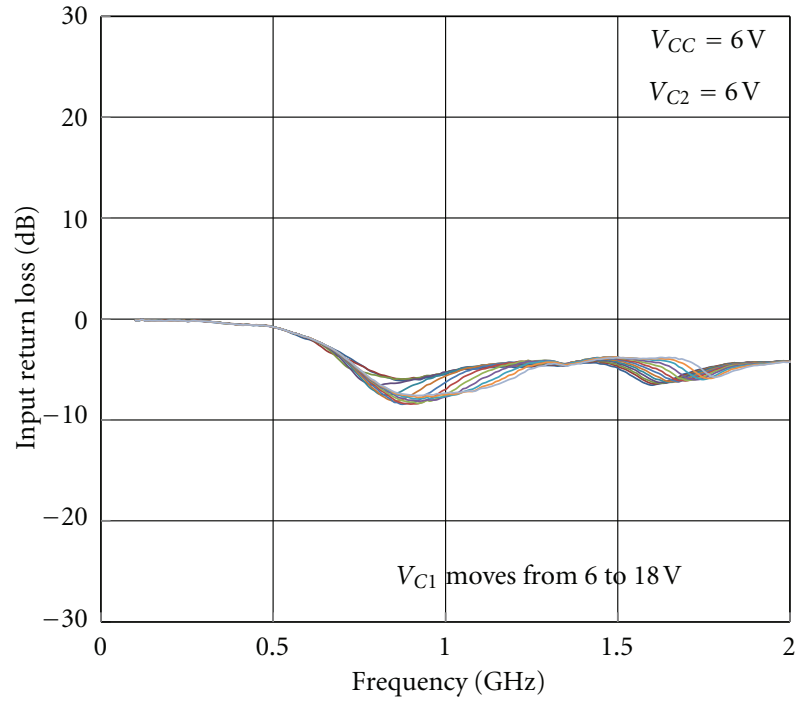

FIGURE 11: Measured input return loss for a fixed $V_{C 2}\left(C_{V 2}\right)$ and a variable $V_{C 1}\left(C_{V 1}\right)$.

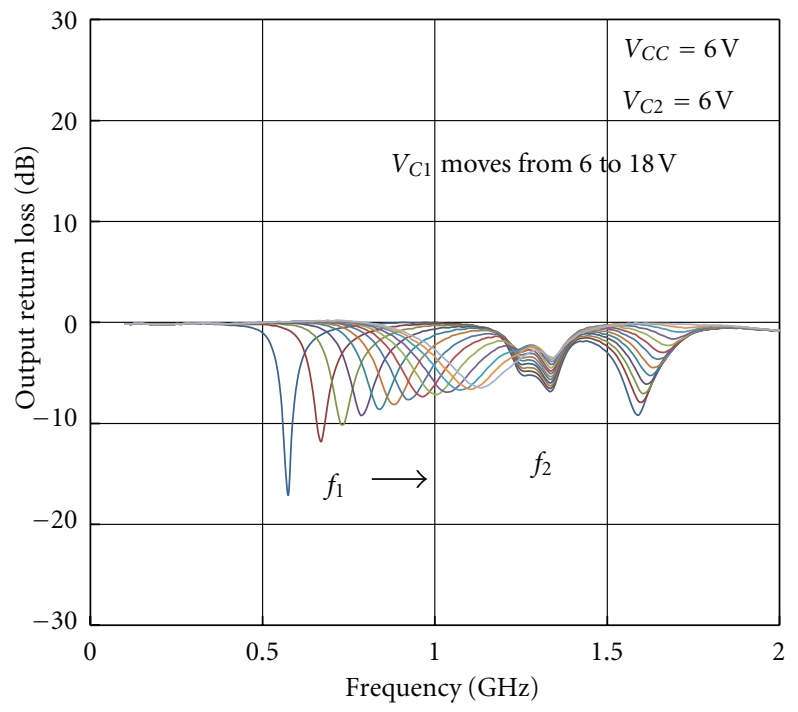

FIGURE 12: Measured output return loss for a fixed $V_{C 2}\left(C_{V 2}\right)$ and a variable $V_{C 1}\left(C_{V 1}\right)$.

better than $4.4 \mathrm{~dB}$ at $0.63 \mathrm{GHz}$ and better than $4.3 \mathrm{~dB}$ at $0.97 \mathrm{GHz}$ in Figure 16. As compared with Figures 7 and 10, a narrow variable range was chosen for $V_{C 1}$ and $V_{C 2}$ since it becomes difficult to show all noise figure data at the same time and make clear the difference. Overall the noise figure performances are not seriously affected by the frequency tuning.

4.4. $\mathrm{IIP}_{3} . \mathrm{IIP}_{3}$ performances were measured with two tones of a $10 \mathrm{MHz}$ separation for a fixed $V_{C 1}(6 \mathrm{~V})$ and a variable $V_{C 2}(6-18 \mathrm{~V})$ as well as a variable $V_{C 1}(6-18 \mathrm{~V})$ and a fixed $V_{C 2}(6 \mathrm{~V}) . V_{C C}$ was $6 \mathrm{~V}$. The amplifier has shown the minimum $\mathrm{IIP}_{3}$ of $-8.7 \mathrm{dBm}$ for a fixed $V_{C 1}(6 \mathrm{~V})$ and a variable $V_{C 2}(6-18 \mathrm{~V})$ as well as $-6.5 \mathrm{dBm}$ for a variable $V_{C 1}$ 


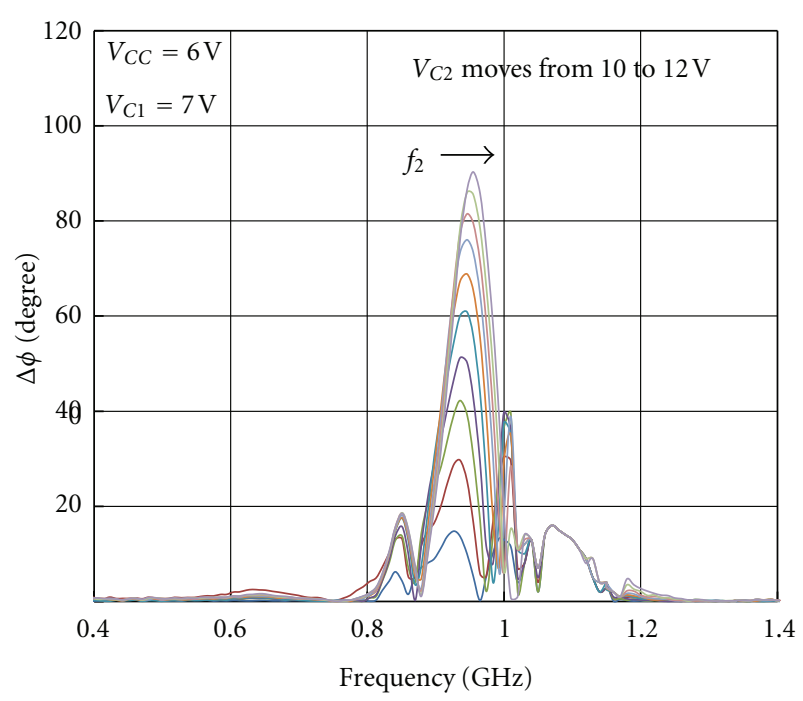

FIgURE 13: Measured phase variations for a fixed $V_{C 1}\left(C_{V 1}\right)$ and a variable $V_{C 2}\left(C_{V 2}\right)$.

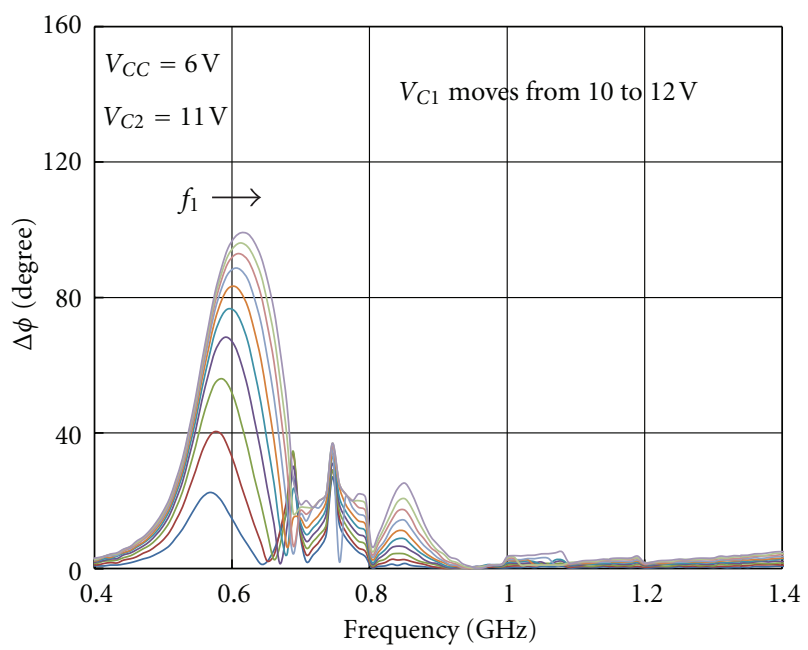

FIgURe 14: Measured phase variations for a fixed $V_{C 2}\left(C_{V 2}\right)$ and a variable $V_{C 1}\left(C_{V 1}\right)$.

$(6-18 \mathrm{~V})$ and a fixed $V_{C 2}(6 \mathrm{~V})$. A total collector current $I_{C}$ appeared in all measurements and ranged from 9 to $12 \mathrm{~mA}$.

\section{Conclusions}

The dual-band SiGe HBT frequency-tunable and phaseshifting differential amplifier has been presented for use in the future active phased array antennas with a multiband, multibeam, and multi-target tracking operation. To realize multifunction capabilities including frequency-tunable and phase-shifting capabilities, the novel design approach to incorporate varactor-loaded, stacked LC resonators with different resonant frequencies into the circuit design has been proposed. Then to make sure the usefulness of the design approach, the dual-band frequency-tunable and phase-shifting differential amplifier using SiGe HBT and Si

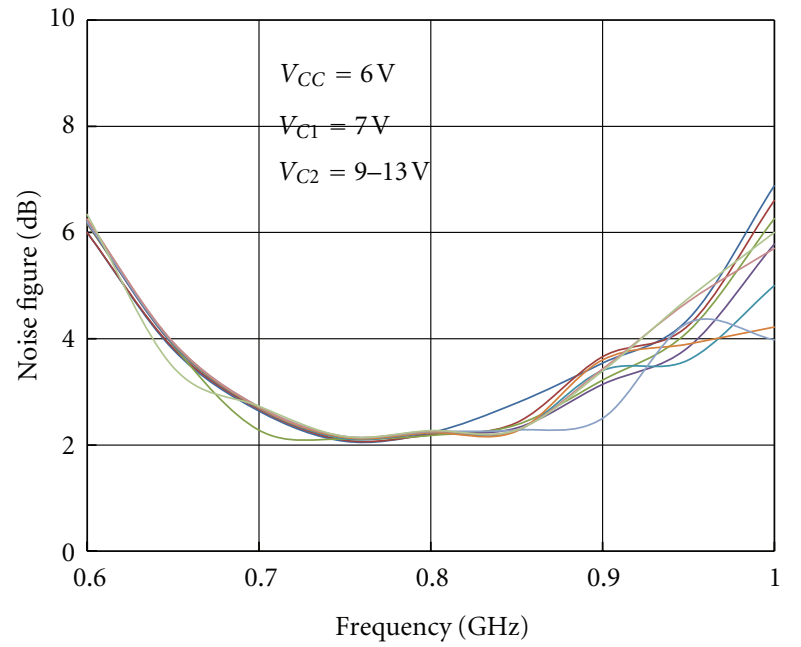

Figure 15: Measured noise figure performances for a fixed $V_{C 1}\left(C_{V 1}\right)$ and a variable $V_{C 2}\left(C_{V 2}\right)$.

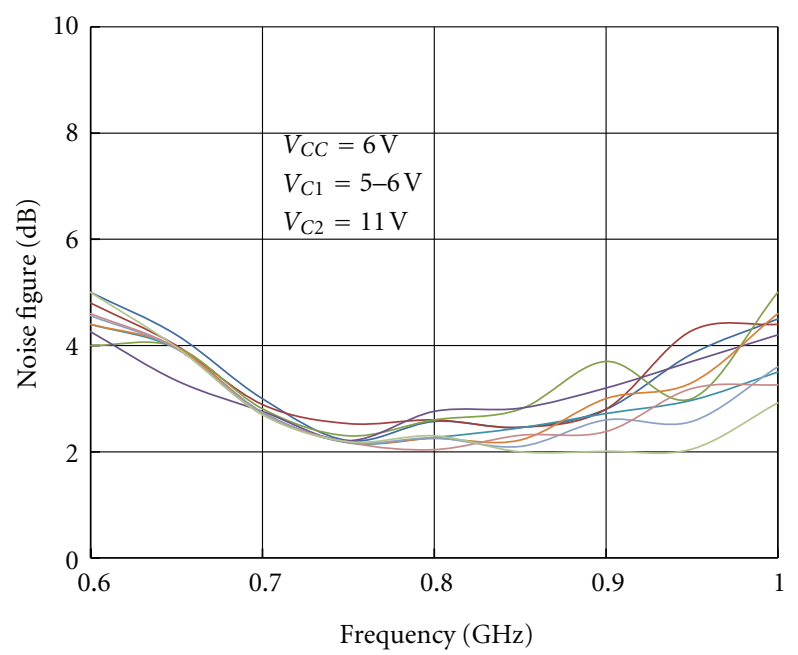

FIGURE 16: Measured noise figure performances for a fixed $V_{C 2}\left(C_{V 2}\right)$ and a variable $V_{C 1}\left(C_{V 1}\right)$.

varactor diode have been actually designed, fabricated, and tested. It is confirmed from the measured results that the design approach proposed in this paper would be one of the candidates for the multifunctional amplifier for use in the current and future wireless, aerospace, microwave, and millimeter-wave sensors and radar systems.

\section{References}

[1] A. R. Rofougaran, M. Rofougaran, and A. Behzad, "Radios for next-generation wireless networks," IEEE Microwave Magazine, vol. 6, no. 1, pp. 38-43, 2005.

[2] R. S. Tahim, "Multi-band antenna technology," in Proceedings of the IEEE Antennas and Propagation Society Symposium, vol. 4, pp. 3968-3971, June 2004. 
[3] H. Hashemi and A. Hajimiri, "Concurrent multiband lownoise amplifiers-theory, design, and applications," IEEE Transactions on Microwave Theory and Techniques, vol. 50, no. 1, pp. 288-301, 2002.

[4] Y. Itoh, "L-Band SiGe HBT differential amplifiers using stacked parallel-resonant circuits," Contemporary Engineering Sciences, vol. 1, no. 3, pp. 127-138, 2008.

[5] M. Shirata, T. Shinohara, M. Sato, and Y. Itoh, "An L-band SiGe HBT differential amplifier with frequency and rejectionlevel tunable, multiple stopband," International Journal of Microwave and Wireless Technologies, vol. 1, no. 4, pp. 285-292, 2009.

[6] C. S. Lin, S. F. Chang, C. C. Chang, and Y. H. Shu, "Design of a reflection-type phase shifter with wide relative phase shift and constant insertion loss," IEEE Transactions on Microwave Theory and Techniques, vol. 55, no. 9, pp. 1862-1868, 2007.

[7] J. C. Wu, T. Y. Chin, S. F. Chang, and C. C. Chang, "2.45-GHz CMOS reflection-type phase-shifter MMICs with minimal loss variation over quadrants of phase-shift range," IEEE Transactions on Microwave Theory and Techniques, vol. 56, no. 10, pp. 2180-2189, 2008.

[8] K. Miyaguchi, M. Hieda, K. Nakahara et al., "An ultra-broadband reflection-type phase-shifter MMIC with series and parallel LC circuits," IEEE Transactions on Microwave Theory and Techniques, vol. 49, no. 12, pp. 2446-2452, 2001.

[9] X. Tang and K. Mouthaan, "Dual-band Class III loaded-line phase shifters," in Proceedings of the Asia-Pacific Microwave Conference (APMC'10), pp. 1731-1734, December 2010.

[10] A. Ocera, E. Sbarra, R. V. Gatti, and R. Sorrentino, "An innovative reconfigurable reflection-type phase shifter for dual band WLAN applications," in Proceedings of the 36th European Microwave Conference (EuMC '06), pp. 64-67, September 2006.

[11] D. R. Banbury, N. Fayyaz, S. Safavi-Naeini, and S. Nikneshan, "A CMOS 5.5/2.4 GHz dual-band smart-antenna transceiver with a novel RF dual-band phase shifter for WLAN 802.11 a/b/g," in Proceedings of the IEEE Radio Frequency Integrated Circuits Symposium (RFIC '04), pp. 157-160, June 2004. 

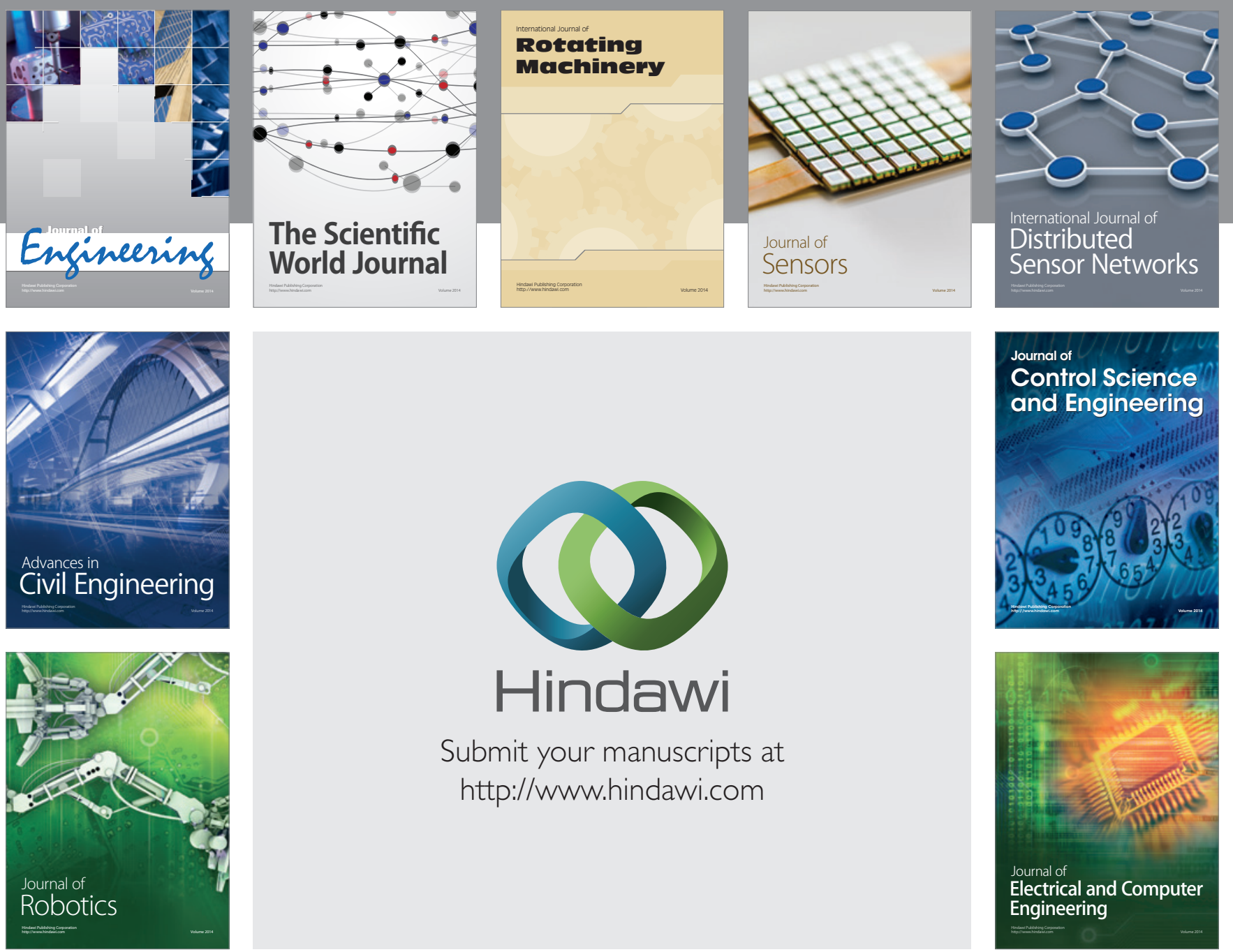

Submit your manuscripts at

http://www.hindawi.com
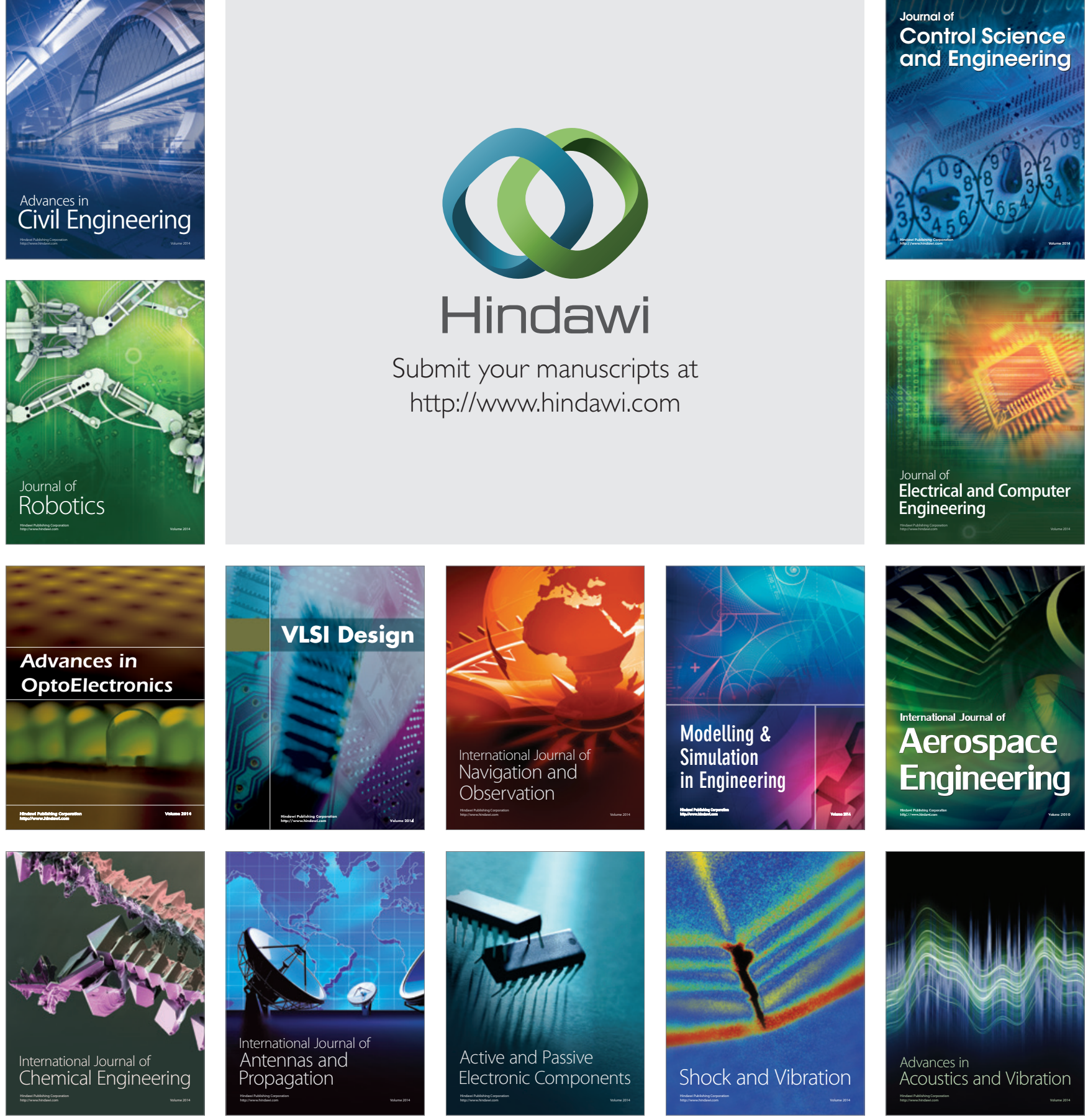